PROCEEDINGS OF THE

AMERICAN MATHEMATICAL SOCIETY

Volume 127, Number 5, Pages 1289-1291

S 0002-9939(99)04816-9

Article electronically published on January 27, 1999

\title{
A SIMPLE PROOF OF A CURIOUS CONGRUENCE BY SUN
}

\author{
ZUN SHAN AND EDWARD T. H. WANG
}

(Communicated by David Rohrlich)

\begin{abstract}
In this note, we give a simple and elementary proof of the following curious congruence which was established by Zhi-Wei Sun:

$$
\sum_{k=1}^{(p-1) / 2} \frac{1}{k \cdot 2^{k}} \equiv \sum_{k=1}^{[3 p / 4]} \frac{(-1)^{k-1}}{k}(\bmod p) .
$$
\end{abstract}

In [4], the following curious congruence for odd prime $p$ was established by ZhiWei Sun:

$$
\sum_{k=1}^{(p-1) / 2} \frac{1}{k \cdot 2^{k}} \equiv \sum_{k=1}^{[3 p / 4]} \frac{(-1)^{k-1}}{k}(\bmod p)
$$

The author's proof, using Pell sequences, is fairly complicated. In fact, a recent article [3] on congruence modulo $p$ ends in the remark that "It seems unlikely that (1) can be proved with the simple approach that we have used here." In the present note, we give a simple and elementary proof of (1). Throughout, $p$ denotes an odd prime.

First of all, it is well known (e.g. [1], [2]) that for $k=0,1,2, \ldots, p-1$,

$$
\left(\begin{array}{c}
p-1 \\
k
\end{array}\right) \equiv(-1)^{k} \quad(\bmod p) .
$$

From (2) we get

$$
\begin{aligned}
\frac{2^{p-1}-1}{2} & =\frac{(1+1)^{p}-2}{2 p}=\frac{1}{2 p} \sum_{k=1}^{p-1}\left(\begin{array}{l}
p \\
k
\end{array}\right)=\frac{1}{2} \sum_{k=1}^{p-1} \frac{1}{k}\left(\begin{array}{l}
p-1 \\
k-1
\end{array}\right) \\
& \equiv \frac{1}{2} \sum_{k=1}^{p-1} \frac{(-1)^{k-1}}{k} \quad(\bmod p) .
\end{aligned}
$$

Received by the editors August 13, 1997.

1991 Mathematics Subject Classification. Primary 11A07, 11A41.

(C)1999 American Mathematical Society 
Let $\varepsilon=e^{\pi i / 4}$. Then

$$
\begin{aligned}
(1+\varepsilon)^{p}+(1-\varepsilon)^{p} & =2+2 \sum_{\substack{1 \leq k \leq p \\
k \text { even }}}\left(\begin{array}{l}
p \\
k
\end{array}\right) \varepsilon^{k} \\
& =2+2 p \sum_{\substack{1 \leq k \leq p \\
k \text { even }}} \frac{1}{k}\left(\begin{array}{l}
p-1 \\
k-1
\end{array}\right) \varepsilon^{k} \\
& \equiv 2-2 p \sum_{\substack{1 \leq k \leq p \\
k \text { even }}} \frac{\varepsilon^{k}}{k}\left(\bmod p^{2}\right) \\
& =2-2 p\left(\sum_{k=1}^{\left[\frac{p-1}{4}\right]} \frac{(-1)^{k}}{4 k}+i \sum_{k=1}^{\left[\frac{p+1}{4}\right]} \frac{(-1)^{k-1}}{4 k-2}\right) \\
& =2-\frac{p}{2} \sum_{k=1}^{\left[\frac{p-1}{4}\right]} \frac{(-1)^{k}}{k}+i p \sum_{k=1}^{\left[\frac{p+1}{4}\right]} \frac{(-1)^{k}}{2 k-1} \\
& =2-\frac{p}{2} A+i p B
\end{aligned}
$$

where

$$
A=\sum_{k=1}^{\left[\frac{p-1}{4}\right]} \frac{(-1)^{k}}{k} \quad \text { and } \quad B=\sum_{k=1}^{\left[\frac{p-1}{4}\right]} \frac{(-1)^{k}}{2 k-1}
$$

Since $\bar{\varepsilon}=\varepsilon^{-1}$, taking modulus of both sides of (4) yields

(5)

$$
\begin{aligned}
4-2 p A & \equiv\left(2-\frac{p}{2} A\right)^{2}+p^{2} B^{2} \\
& \equiv 4-2 p A \equiv\left((1+\varepsilon)^{p}+(1-\varepsilon)^{p}\right)\left(\left(1+\varepsilon^{-1}\right)^{p}+\left(1-\varepsilon^{-1}\right)^{p}\right) \\
& =\left(2+\varepsilon+\varepsilon^{-1}\right)^{p}+\left(2-\varepsilon-\varepsilon^{-1}\right)^{p} \\
& =(2+\sqrt{2})^{p}+(2-\sqrt{2})^{p} \\
& =2^{p+1}+2 \sum_{\substack{1 \leq k \leq p \\
k \text { even }}}\left(\begin{array}{l}
p \\
k
\end{array}\right) 2^{p-k}(\sqrt{2})^{k} \\
& =2^{p+1}+2^{p+1} \sum_{k=1}^{(p-1) / 2}\left(\begin{array}{c}
p \\
2 k
\end{array}\right) \frac{1}{2^{k}} \\
& =2^{p+1}+2^{p} p \sum_{k=1}^{(p-1) / 2} \frac{1}{k \cdot 2^{k}}\left(\begin{array}{c}
p-1 \\
2 k-1
\end{array}\right) \\
& \equiv 2^{p+1}-2^{p} p \sum_{k=1}^{(p-1) / 2} \frac{1}{k \cdot 2^{k}}\left(\bmod p^{2}\right) .
\end{aligned}
$$


From $(5)$ and $(3)$ we obtain, since $2^{p-1} \equiv 1(\bmod p)$,

$$
\begin{aligned}
A & \equiv-\frac{2^{p}-2}{p}+2^{p-1} \sum_{k=1}^{(p-1) / 2} \frac{1}{k \cdot 2^{k}} \\
& \equiv \sum_{k=1}^{p-1} \frac{(-1)^{k}}{k}+\sum_{k=1}^{(p-1) / 2} \frac{1}{k \cdot 2^{k}}(\bmod p)
\end{aligned}
$$

and so

$$
\begin{aligned}
\sum_{k=1}^{(p-1) / 2} \frac{1}{k \cdot 2^{k}} & \equiv-\sum_{k=1}^{p-1} \frac{(-1)^{k}}{k}+A=\sum_{k=1}^{p-1} \frac{(-1)^{k-1}}{k}+\sum_{k=1}^{\left[\frac{p-1}{4}\right]} \frac{(-1)^{k}}{k} \\
& =\sum_{k=1}^{p-1} \frac{(-1)^{k-1}}{k}+\sum_{k=p-\left[\frac{p-1}{4}\right]}^{p-1} \frac{(-1)^{p-k}}{p-k} \\
& \equiv \sum_{k=1}^{p-1} \frac{(-1)^{k-1}}{k}-\sum_{k=p-\left[\frac{p-1}{4}\right]}^{p-1} \frac{(-1)^{k-1}}{k}(\bmod p) \\
& =\sum_{k=1}^{\left[\frac{3 p}{4}\right]} \frac{(-1)^{k-1}}{k}
\end{aligned}
$$

and (1) is proved.

\section{ACKNOWLEDGEMENT}

This paper was written when the first author was visiting the Mathematics Department of Wilfrid Laurier University, December 1996-August 1997. The hospitality of Wilfrid Laurier University is greatly appreciated.

\section{REFERENCES}

[1] Louis Comet, Advanced Combinatorics, D. Reidel Publishing Company, 1974.

[2] G. H. Hardy and E. M. Wright, An Introduction to the Theory of Numbers, Fourth Edition, Clarendon Press, Oxford, 1960.

[3] Winfried Kohnen, A simple congruence modulo p, Amer. Math. Monthly 104 (1997), 444445. MR 98e: 11004

[4] Zhi-Wei Sun, A congruence for primes, Proc. Amer. Math. Soc. 123 (1995), 1341-1346. MR 95f: 11003

Department of Mathematics, Nanjing Normal University, Nanjing, Jiangsu, 210097, People's Republic of China

Department of Mathematics, Wilfrid Laurier University, Waterloo, Ontario, Canada N2L $3 \mathrm{C} 5$

E-mail address: ewang@machl.wlu.ca 